\title{
End-to-End Average BER in Multihop Wireless Networks over Fading Channels
}

\author{
Eduardo Morgado, Inmaculada Mora-Jiménez, Juan J. Vinagre, Javier Ramos, and Antonio J. Caamaño
}

\begin{abstract}
This paper addresses the problem of finding an analytical expression for the end-to-end Average Bit Error Rate (ABER) in multihop Decode-and-Forward (DAF) routes within the context of wireless networks. We provide an analytical recursive expression for the most generic case of any number of hops and any single-hop ABER for every hop in the route. Then, we solve the recursive relationship in two scenarios to obtain simple expressions for the end-to-end ABER, namely: (a) The simplest case, where all the relay channels have identical statistical behaviour; (b) The most general case, where every relay channel has a different statistical behaviour. Along with the theoretical proofs, we test our results against simulations. We then use the previous results to obtain closed analytical expressions for the end-to-end ABER considering DAF relays over Nakagami- $m$ fading channels and with various modulation schemes. We compare these results with the corresponding expressions for Amplify-and-Forward (AAF) and, after corroborating the theoretical results with simulations, we conclude that DAF strategy is more advantageous than the AAF over Nakagami- $m$ fading channels as both the number of relays and $m$-index increase.
\end{abstract}

Index Terms-Bit error rate, end-to-end performance, decode and forward, amplify and forward, fading channel, multihop wireless networks.

\section{INTRODUCTION}

$\mathbf{M}$ ULTIHOP Wireless Networks (MHWN) have been the focus of an intense research effort in recent times [1], [2]. Their main advantages with respect to wireless direct transmission between source and destination are, on the one hand, the increase of coverage and battery life and, on the other hand, the reduction in the interference level. In spite of the increasing interest on MHWN, specially with the development of the cooperative or collaborative wireless networks [3], [4], the inherent complexity of these communication systems makes it very difficult to evaluate their performance in a general framework [5], [6]. In fact, to the best of our knowledge, the theoretical comparison of the end-to-end Average Bit Error Rate (ABER) between the Amplify-and-Forward (AAF) approach (analog relaying) [7], [8] and the Decodeand-Forward (DAF) strategy (digital relaying) [9]-[11] has just been exactly solved for the particular case of routes with two consecutive hops [12]. Following this line, in this paper we

Manuscript received February 16, 2009; revised October 30, 2009 and March 10, 2010; accepted June 10, 2010. The associate editor coordinating the review of this paper and approving it for publication was R. Nabar.

The authors are with the Signal Theory and Communications Department, Rey Juan Carlos University of Madrid, Camino del Molino, s/n, 28943, Fuenlabrada, Spain (e-mail: \{eduardo.morgado, inmaculada.mora, juanjose.vinagre, javier.ramos, antonio.caamano\}@urjc.es).

This work has been supported by Research Project TEC2009-12098 from the Spanish Ministry of Science and Innovation (MICINN).

Digital Object Identifier 10.1109/TWC.2010.070710.090240

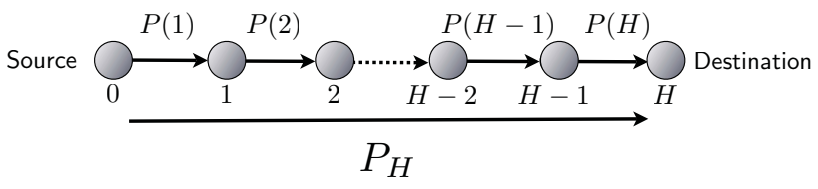

Fig. 1. Multihop route with $H+1$ nodes. The single-hop ABER from node $i$ to node $i+1$ is represented by $P(i+1)$. The end-to-end ABER from node 0 to node $H$ is represented by $P_{H}$.

analytically solve the theoretical calculation of the end-to-end ABER in a scenario with an arbitrary number of consecutive hops, suffering from multipath fading and using the DAF strategy. Both the case of identical and distinct consecutive channels in the route are solved, proving also the invariance of the end-to-end ABER to the ordering of the distinct channels. Using these results, we are able to compare the DAF and AAF strategies for different wireless fading channels and different modulations. These results allows us to analytically obtain the diversity gain of DAF for routes with an arbitrary number of hops.

The remaining of this paper is organized as follows. Section II introduces the scenario under consideration and formulates an analytical recursive expression for the most generic case, i.e., any number of hops and any single-hop ABER in multihop DAF routes. This Section also provides the end-to-end ABER for two opposed scenarios where all the relay channels have, respectively, identical and different statistical behaviour. Section III studies the effect of the fading channel and its severity along with the modulation on the ABER for both DAF and AAF strategies. Furthermore, we are able to provide the analytic diversity gain for Nakagami- $m$ channels. Finally, Section IV summarizes the main results of the paper.

\section{End-to-End Average BER in a Multihop WIRELESS NETWORK}

\section{A. Problem Statement}

Let us consider a route with $H+1$ nodes in a MHWN with DAF relaying strategy. Fig. 1 shows the considered scheme, where the node labelled as 0 corresponds to the source node and the node labelled as $H$ corresponds to the destination node.

Considering this scenario and index $h \in\{1,2, \ldots, H\}$, we define $P(h)$ as the single-hop ABER from node $h-1$ to node $h$, and $P_{h}$ as the end-to-end ABER from source node (node $0)$ to node $h$. Taking into account these definitions, we can express the probability of correct bit transmission from source 
node to node $h-1$ as $\left(1-P_{h-1}\right)$, and the probability of correct bit transmission from node $h-1$ to node $h$ as $(1-P(h))$.

Assuming statistical independence among the channels of successive hops, the end-to-end error probability of a binary symbol that experiences $H \geq 1$ successive transmissions, $P_{H}$, can be recursively expressed as

$$
P_{H}=\left(1-P_{H-1}\right) P(H)+P_{H-1}(1-P(H))
$$

where the first term accounts for the joint probability of a correct bit transmission from the source node up to the nextto-last node in the whole transmission route and a wrong last transmission, and the second term accounts for the joint probability of a wrong bit transmission from the source node up to the second last node and a correct last transmission. The initial condition for (1) is given by $P_{0}=0$.

Note that (1) takes into account that, in a multihop network, a correct bit transmission from node $i$ to node $j$ (with $j \geq$ $i+2$ ) is equivalent to an even number of wrong single-hop bit transmissions (bit value changes) between nodes $i$ and $j$. Following the same reasoning, a wrong bit transmission from node $i$ to node $j$ is equivalent to an odd number of wrong single-hop bit transmission between both nodes. If Average Symbol Error Rate (ASER) was to be analyzed and highorder modulation schemes were to be used, there would be multiple combinations of flawed retransmissions that would provide error-free symbol transmissions. As a consequence, although the analysis of the end-to-end ABER based on either single-hop ABER or single-hop ASER are equivalent, from an analytical point of view, the former approach is considerably simpler than the latter.

\section{B. Single-Hops with Identical Statistical Behaviour}

In this section we study the simplest scenario in a multihop network where all the single-hops in the route have the same statistical behaviour, i.e., all the links are i.i.d with equal average received Signal to Noise Ratio (SNR). Therefore,

$$
P(h)=P_{\text {hop }}, \quad h \in\{1,2, \ldots, H\} .
$$

This simplification is frequently assumed in the literature [1], [2] when the single-hop ABER is unknown and therefore $P_{\text {hop }}$ is obtained according to the channel statistical distribution. This simplification is also advantageous in order to achieve a compact expression for the end-to-end ABER, $P_{H}$, just dependent on both $P_{\text {hop }}$ and number of hops $H$.

Theorem 1: Let a bit be transmitted by regenerating nodes from source node 0 to destination node $H$ through $H$ channels with identical statistical behaviour and characterized by the single-hop ABER $P(h)=P_{\text {hop }}$, with $h \in\{1,2, \ldots, H\}$. Then, the probability that the received bit in node $H$ is different from the transmitted bit by node 0 is given by

$$
P_{H}=\frac{1}{2}\left(1-\left(1-2 P_{\mathrm{hop}}\right)^{H}\right), \forall H \geq 1 .
$$

Proof: See Appendix A.

Usually, the expression used to determine the end-to-end ABER for routes with the same value of single-hop ABER has been [2]

$$
P_{H}=1-\left(1-P_{\mathrm{hop}}\right)^{H} .
$$

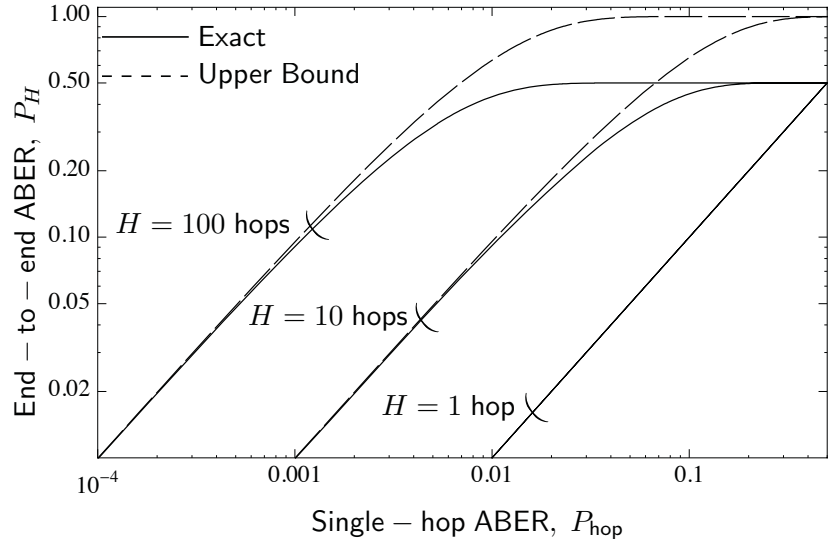

Fig. 2. End-to-end ABER for $H=1,10$ and 100 statistically identical relays. Continuous line corresponds to the exact value of $P_{H}$ given by (3), and dashed line corresponds to its upper bound (4).

A comparison between (3) and (4) is shown in the log$\log$ representation of Fig. 2 for $H=1,10$ and 100. For a fixed $P_{\text {hop }}$, we can observe remarkable differences as $H$ increases. In fact, (4) is an upper-bound to the exact value of $P_{H}$ because (4) simply accumulates the errors through the relays, not taking into account that, in a DAF scheme, an even number of wrong single-hop transmissions in the route results in a correct end-to-end transmission.

On the other hand, for a fixed $H$ and $P_{\text {hop }}$ low enough, we observe that (4) is an accurate approximation to (3). In this case, Fig. 2 shows a linear behaviour for $P_{H}$. In order to find a simple approximation to (3) proving its linear behaviour when $P_{\text {hop }}$ is low enough, we may expand (3) as

$$
P_{H}=\left(\begin{array}{c}
H \\
1
\end{array}\right) P_{\text {hop }}-2\left(\begin{array}{c}
H \\
2
\end{array}\right) P_{\text {hop }}^{2}+\ldots+(-2)^{H-1}\left(\begin{array}{c}
H \\
H
\end{array}\right) P_{\text {hop }}^{H}
$$

Then, since $P_{\text {hop }}$ is low enough, (5) can be written retaining only the leading term as

$$
P_{H} \approx H P_{\mathrm{hop}},
$$

and the linear behaviour of $P_{H}$ with regenerative relays is analytically proved. Using (6) and defining $\delta \equiv \log _{10} H$ and $\beta \equiv-\log _{10} P_{\text {hop }}$, the resulting end-to-end ABER can be simply written as

$$
P_{H} \approx 10^{-(\beta-\delta)} .
$$

Additionally, from Fig. 2, note that there are remarkable differences between (3) and (4) only when $(\beta-\delta) \leq 1$ and $\delta>0$. This observation let us to conclude that a low enough $P_{\text {hop }}$ for a fixed $H>1$ is equivalent to $(\beta-\delta) \leq 1$.

Since (3) is valid irrespective of the radio channel and the bit error rate for any modulation, to check our theoretical results through simulation, we will consider a Rayleigh fading channel and a Binary Phase-Shift Keying (BPSK) modulation scheme. Assuming the same statistical performance for all links in the multihop route, and using the single-hop ABER for BPSK given in [13, eq. (8.104)], the exact end-to-end ABER 


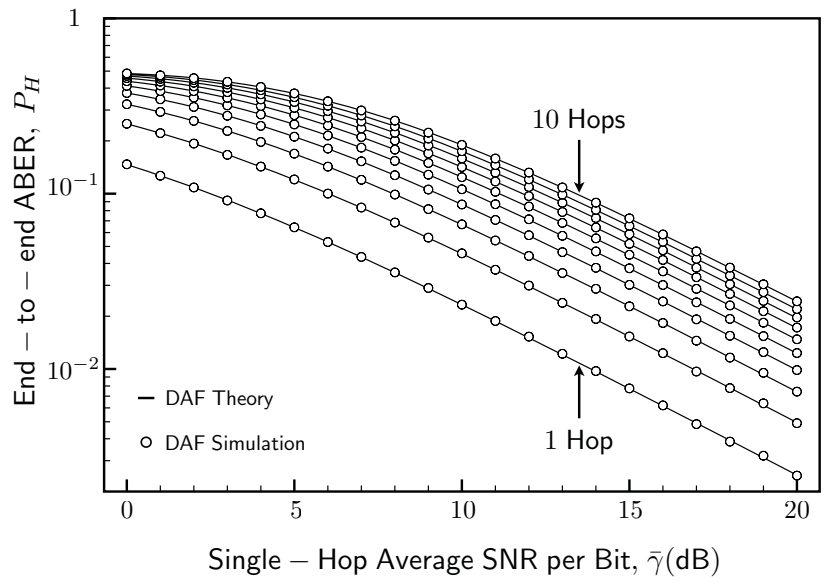

Fig. 3. End-to-end ABER for $H=1$ to $H=10$ with statistically identical relays over Rayleigh fading channels using BPSK modulation. Theoretical (8) and simulation results are plotted with continuous lines and circles, respectively.

of the $H$-hop route can be written as

$$
P_{H}=\frac{1}{2}\left(1-\left(\frac{\bar{\gamma}}{1+\bar{\gamma}}\right)^{H / 2}\right)
$$

where the average single-hop SNR per bit is given by $\bar{\gamma}=$ $\bar{\alpha}^{2} E_{b} / N_{0}$, with $\alpha^{2}$ being the mean square value of the random variable (r.v.) corresponding to the fading amplitude, $E_{b}$ the mean energy of the transmitted bits and $N_{0}$ the power spectral density of thermal noise. Fig. 3 shows both the analytical results according to (8) and the experimental results with $10^{6}$ Monte Carlo simulations for each different pair of values of $H$ and $\bar{\gamma}$. Note that the experiments are consistent with the theoretical results. Therefore, (3) represents the exact end-toend ABER of multihop routes with homogeneous channels.

In addition, we can use (3) to analyze the increase of the end-to-end ABER with increasing number of hops. Moreover, we can define the end-to-end SNR of a route as the SNR of a single-hop with ABER equal to the end-to-end ABER of the considered route. According to this, Fig. 4 corroborates the linear increase of the end-to-end ABER and the linear decrease of the end-to-end SNR with increasing number of hops.

\section{Single-Hops with Different Statistical Behaviour}

To extend our results in the previous section, we now consider the most general case of different statistical behaviour for every single-hop.

Theorem 2: Let a bit be transmitted by regenerating nodes from source node 0 to destination node $H$ through $H$ channels with different statistical behaviour and characterized by the single-hop ABER $P(h)$, with $h \in\{1,2, \ldots, H\}$. Then, the probability that the received bit in node $H$ is different from the transmitted bit by node 0 is given by

$$
P_{H}=\sum_{i=1}^{H}\left(P(i) \prod_{j=i+1}^{H}(1-2 P(j))\right)
$$

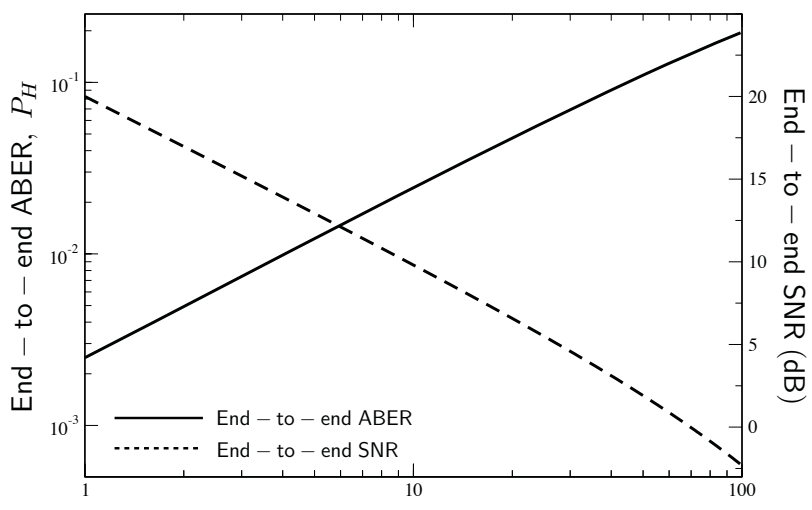

Number of Hops, $H$

Fig. 4. End-to-end ABER and end-to-end SNR for $H=1$ to $H=100$ with statistically identical relays over Rayleigh fading channels using BPSK modulation. The single-hop average SNR per bit in every hop is $\bar{\gamma}=20 \mathrm{~dB}$.

Proof: See Appendix B.

Corollary 1: Let a bit be transmitted by regenerating nodes from source node 0 to destination node $H$, through $H$ channels with different statistical behaviour and characterized by the single-hop ABER $P(h)$, with $h \in\{1,2, \ldots, H\}$. Then, the probability that the received bit in node $H$ is different from the transmitted bit by node 0 does not depend on the order of the single-hops.

Proof: See Appendix C.

Corollary 1 allows us to study the extension of (8) to the general case where the $H$ hops in the route can be classified into $N$ kinds of single hops, each one characterized by its corresponding average SNR per bit, $\bar{\gamma}_{n}$ with $n \in\{1,2, \ldots, N\}$. Thus, assuming that $H_{n}$ is the number of single hops of the $n-t h$ type, i.e. $\sum_{n=1}^{N} H_{n}=H$, we obtain from (9) the endto-end ABER considering BPSK modulation over Rayleigh fading channels

$$
P_{H}=\frac{1}{2}-\frac{1}{2} \prod_{n=1}^{N}\left(\frac{\bar{\gamma}_{n}}{1+\bar{\gamma}_{n}}\right)^{H_{n} / 2} .
$$

Analogous to the analysis in the previous section, we examine a case of interest in the high SNR region. For end-to-end ABER values where (6) becomes valid, we can carry out a simplified analysis of the correlation between the end-to-end ABER with the ABER of the link with the lowest average SNR. For example, in the extreme case of a route with $H$ hops where there are $H-1$ hops with good average SNR (with $P_{\text {Class } 1}, \delta_{\text {Class } 1} \equiv \log _{10}(H-1)$ and $\left.\beta_{\text {Class } 1} \equiv-\log _{10} P_{\text {Class } 1}\right)$ and only one hop with low average SNR (with $P_{\text {Class } 2}$ and $\beta_{\text {Class } 2} \equiv-\log _{10} P_{\text {Class } 2) \text {, this worst }}$ link is determinant in the calculation of the end-to-end ABER when $\beta_{\text {Class } 2}<\left(\beta_{\text {Class } 1}-\delta_{\text {Class } 1}\right)$ or, in an equivalent form, when

$$
\frac{P_{\text {Class } 2}}{P_{\text {Class } 1}}>H-1 \text {. }
$$

A general validation for the case where different channels are involved in the consecutive hops in a route requires an infinite number of different mixtures. Therefore, we select a 


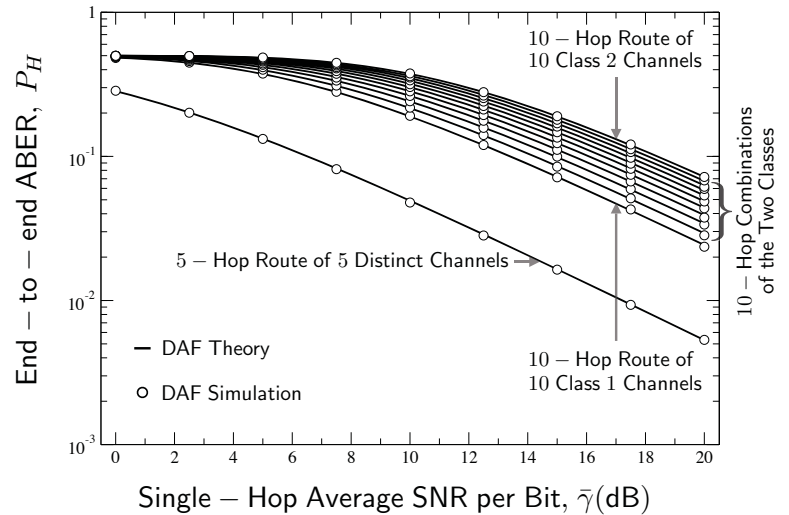

Fig. 5. End-to-end ABER for routes with different relays over Rayleigh fading channels using BPSK modulation. Theoretical (10) and simulation results are plotted with continuous lines and circles, respectively. The first line from the bottom represent the results for a route of 5 hops, with the first hop characterized by the single-hop average SNR $\bar{\gamma}_{1}=\bar{\gamma} \mathrm{dB}$ and the rest of hops, from $i=2$ to $i=5$, characterized by $\bar{\gamma}_{i}=\bar{\gamma}+2 i-1 \mathrm{~dB}$. The higher curves correspond to routes of 10 hops, with each route composed of two classes of single-hop channels: Class 1 , determined by $\bar{\gamma}_{1}=\bar{\gamma}$; and Class 2 , with $\bar{\gamma}_{2}=\bar{\gamma}-5 \mathrm{~dB}$. These eleven routes are respectively composed (from bottom to top) of $10-n$ single-hops of Class 1 and $n$ single-hops of Class 2 , where $n=\{0, \ldots, 10\}$.

few representative examples for the validation of our theoretic results through simulation. Fig. 5 shows the theoretical and simulation results of the end-to-end ABER for routes composed of different single-hop average SNR per bit and different number of hops with BPSK modulation and Rayleigh fading channels. The first case to be considered is that of a route with 5 hops, with the first hop characterized by the single-hop average SNR $\bar{\gamma}_{1}=\bar{\gamma} \mathrm{dB}$ and the remaining, from $i=2$ to $i=5$, by $\bar{\gamma}_{i}=\bar{\gamma}+2 i-1 \mathrm{~dB}$. The second case corresponds to routes of 10 hops, with each route composed of the aforementioned two classes of single-hop channels: Class 1 , determined by $\bar{\gamma}_{1}=\bar{\gamma}$; and Class 2 , with $\bar{\gamma}_{2}=\bar{\gamma}-5 \mathrm{~dB}$. These eleven routes are respectively composed of $(10-n)$ single-hops of Class 1 and $n$ single-hops of Class 2, where $n=\{0, \ldots, 10\}$. The consistency between theory and simulation is complete in both cases. Thus, (9) represents the exact end-to-end ABER of multihop routes with heterogeneous channels.

\section{Analog Versus Digital Relaying over Fading CHANNELS}

In order to determine whether it is preferable to use multihop analog relaying $(A A F)$ or perform a digital regeneration of the original signal in each stage of the relaying (DAF) [1], [12], [14], in this section we evaluate the end-to-end ABER of both strategies in MHWN over Nakagami- $m$ fading channels using the theoretical results achieved in Section II-B.

Our motivation for considering the Nakagami- $m$ distribution rests in its capability to model a wide range of multipath fading channels through different values of the Nakagami fading parameter $m$ [13]. Thus, the fading effect when there is no direct line of sight (LOS) path is accurately modelled by the Rayleigh distribution, which can be obtained as a special case of Nakagami- $m$ for $m=1$. Fading environments less severe than those of Rayleigh fading are also considered by Nakagami- $m$ distribution when $m>1$, approximating others such as the Rice fading (used to model the effect of one strong direct LOS component and many random weaker components). Additionally, the Nakagami- $m$ distribution is of interest because it can represent the best fit to landmobile and indoor mobile multipath propagation, scintillating ionospheric radio links, and satellite-to-indoor and satellite-tooutdoor radio wave propagation [15].

\section{A. Nakagami-m Fading}

In Section II we have proposed an analytical expression for DAF relaying with an arbitrary number of relay-nodes and an arbitrary single-hop ABER. Now, to compare DAF and AAF strategies, we need an analytical expression for the end-to-end ABER using AAF relaying. To the best of our knowledge, no analytical expression for the general case of $H$-hops with AAF relaying is available, and just the simplest case of two hops $(H=2)$ for AAF with fading has been solved [14]. The only further result, regarding the case of an arbitrary number of hop and AAF, is the Lower Bound for the end-to-end ABER [12]. We will use these and our results to evaluate the impact of the modulation scheme in the end-to-end ABER with DAF and AAF strategies.

We will proceed first with the AAF analysis. As in [13], we resort to the approach based on the Moment Generating Function (MGF) to obtain the analytic formulation of the endto-end ABER for MHWN with AAF relaying. We define the MGF associated with a semi-definite positive r.v. $\gamma$ as

$$
M_{\gamma}(s)=\int_{0}^{\infty} p(\gamma) e^{-s \gamma} d \gamma
$$

where $p(\gamma)$ is the probability density function of r.v. $\gamma$.

In the simplest case of two links with the same statistical behaviour, characterized by the average SNR $(\bar{\gamma})$, the Nakagami$m$ MGF can be written as [16, eq. (26)]

$$
M_{\gamma}(s, m, H=2)={ }_{2} F_{1}\left(m, 2 m ; m+\frac{1}{2} ;-s \frac{\bar{\gamma}}{4 m}\right)
$$

where ${ }_{2} F_{1}(a, b ; c, z)$ is the Gauss Hypergeometric function $[17$, eq. (9.100)].

To study the influence of the fading parameter $m$ on the endto-end ABER, we will consider the 4-Quadrature Amplitude Modulation (4-QAM) scheme. Thus, for 4-QAM and $H=2$ hops, the corresponding end-to-end ABER can be obtained through [13, eq. (8.111)] as

$$
\begin{gathered}
P_{4-\operatorname{QAM}}^{\mathrm{AAF}}(\bar{\gamma}, m, H=2) \cong \\
\cong \frac{1}{\pi} \int_{0}^{\pi / 2} M_{\gamma}\left(\frac{1}{\sin ^{2} \theta}, m, H=2\right) d \theta= \\
=\frac{1}{2}-\frac{2^{1-8 m} \pi^{3 / 2} \Gamma(4 m)}{\Gamma^{3}(m)} \sqrt{\frac{\bar{\gamma}}{m}} \times \\
\times{ }_{3} \tilde{F}_{2}\left(m+\frac{1}{2}, 2 m+\frac{1}{2}, \frac{1}{2} ; m+1, \frac{3}{2} ;-\frac{\bar{\gamma}}{4 m}\right)
\end{gathered}
$$


where $\Gamma(\cdot)$ is the Gamma function and

$$
\begin{aligned}
& { }_{p} \tilde{F}_{q}\left(a_{1}, a_{2}, \ldots, a_{p} ; b_{1}, b_{2}, \ldots, b_{q} ; z\right) \equiv \\
\equiv & \frac{{ }_{p} F_{q}\left(a_{1}, a_{2}, \ldots, a_{p} ; b_{1}, b_{2}, \ldots, b_{q} ; z\right)}{\Gamma\left(b_{1}\right) \Gamma\left(b_{2}\right) \cdots \Gamma\left(b_{q}\right)}
\end{aligned}
$$

with ${ }_{p} F_{q}\left(a_{1}, a_{2}, \ldots, a_{p} ; b_{1}, b_{2}, \ldots, b_{q} ; z\right)$ being the Generalized Hypergeometric Function [17, Eq. (9.14.1)].

In the DAF case, using the MGF for the single-hop Nakagami- $m$ channel $[13$, p. 21$]$

$$
M_{\gamma}^{\mathrm{DAF}}(s, m, H=1)=\left(1+s \frac{\bar{\gamma}}{m}\right)^{-m}
$$

we can evaluate its single-hop ABER for 4-QAM modulation through [13, eq. (8.111)]

$$
\begin{gathered}
P_{4-\mathrm{QAM}}^{\mathrm{DAF}}(\bar{\gamma}, m, H=1) \cong \\
\cong \frac{1}{\pi} \int_{0}^{\pi / 2} M_{\gamma}\left(\frac{1}{\sin ^{2} \theta}, m, H=1\right) d \theta= \\
=\left(\frac{\bar{\gamma}}{m}\right)^{-m} \frac{\Gamma\left(m+\frac{1}{2}\right)}{2 \sqrt{\pi}}{ }_{2} \tilde{F}_{1}\left(m, m+\frac{1}{2} ; m+1 ;-\frac{m}{\bar{\gamma}}\right) .
\end{gathered}
$$

Finally, to obtain the end-to-end ABER for a route of $H$ hops, we insert (17) into (3)

$$
\begin{gathered}
P_{4-\mathrm{QAAM}}^{\mathrm{DAF}}(\bar{\gamma}, m, H) \cong \\
\cong \frac{1}{2}-\frac{1}{2}\left(1-\left(\frac{\bar{\gamma}}{m}\right)^{-m} \frac{\Gamma\left(m+\frac{1}{2}\right)}{\sqrt{\pi}} \times\right. \\
\left.\times{ }_{2} \tilde{F}_{1}\left(m, m+\frac{1}{2} ; m+1 ;-\frac{m}{\bar{\gamma}}\right)\right)^{H} .
\end{gathered}
$$

To complete our analysis, we will focus on a particular case of the Nakagami- $m$ fading channel and we will study the influence of the modulation technique on the end-to-end ABER for the AAF and DAF strategies and that particular channel. Specifically, for simplicity of the corresponding MGF, we have chosen the Rayleigh fading model and the Binary Differential Phase-Shift Keying (BDPSK) modulation technique (associated to a differentially coherent detection, instead of the ideal coherent detection of 4-QAM).

Starting with BDPSK modulation and AAF relaying with $H=2$ hops, we use [13, Eq. (8.201)] and (13) to get the following expression for the end-to-end ABER

$$
\begin{gathered}
P_{\mathrm{BDPSK}}^{\mathrm{AAF}}(\bar{\gamma}, m=1, H=2)= \\
=\frac{1}{2} M_{\gamma}(s=1, m=1, H=2)=\frac{1}{2}{ }_{2} F_{1}\left(1,2 ; \frac{3}{2},-\frac{\bar{\gamma}}{4}\right) .
\end{gathered}
$$

For 4-QAM modulation, AAF relaying and the same number of $H=2$ hops, the end-to-end ABER is obtained from (14) as

$$
\begin{aligned}
& P_{4-\mathrm{QAM}}^{\mathrm{AAF}}(\bar{\gamma}, m=1, H=2) \cong \\
\cong & \frac{1}{2}-\frac{3}{32} \pi \sqrt{\bar{\gamma}}{ }_{2} F_{1}\left(\frac{1}{2}, \frac{5}{2} ; 2,-\frac{\bar{\gamma}}{4}\right) .
\end{aligned}
$$

Though no general expression for the end-to-end ABER for an $H$-hop route with AAF relaying is known, Hasna [12] has found an Upper Bound for the equivalent end-to-end SNR with Rayleigh fading, leading to the following Lower Bound for the MGF

$$
M_{\gamma}^{\mathrm{LB}}(s, m=1, H)=\frac{1}{1+s\left[\sum_{h=1}^{H} \frac{1}{\bar{\gamma}_{h}}\right]^{-1}}
$$

where $\bar{\gamma}_{h}$ is the average SNR per bit for the $h$-th AAF relay. Assuming all single-hops have identical statistical behaviour, $\bar{\gamma}_{h}=\bar{\gamma}$ with $h \in\{1, \ldots, H\}$, and using (21) we obtain the following Lower Bound for the end-to-end ABER with BDPSK modulation

$$
\begin{gathered}
P_{\mathrm{BDPSK}}^{\mathrm{AAF}}(\bar{\gamma}, m=1, H) \geq \\
\geq \frac{1}{2} M_{\gamma}^{\mathrm{LB}}(s=1, m=1, H)=\frac{1}{2} \frac{H}{H+\bar{\gamma}}
\end{gathered}
$$

and with 4-QAM, the Lower Bound for the end-to-end ABER can be written as

$$
\begin{gathered}
\geq \frac{1}{\pi} \int_{0}^{P_{4-\mathrm{QAM}} \mathrm{AAF}} \frac{1}{1+\frac{1}{\sin ^{2} \theta}\left[\sum_{h=1}^{H} \frac{1}{\bar{\gamma}}\right]^{-1}} d \theta= \\
=\frac{H}{2(H+\bar{\gamma}+\sqrt{\bar{\gamma}(H+\bar{\gamma})})} .
\end{gathered}
$$

We proceed now with DAF relaying to obtain the analytical expressions for the end-to-end ABER using (3). For this purpose, we only need to replace $P_{\text {hop }}$ in (3) with the singlehop ABER for BDPSK modulation [13, eq. (8.207)], obtaining the final expression

$$
P_{\mathrm{BDPSK}}^{\mathrm{DAF}}(\bar{\gamma}, m=1, H)=\frac{1}{2}\left(1-\left(\frac{\bar{\gamma}}{1+\bar{\gamma}}\right)^{H}\right) .
$$

Repeating the same procedure with the single-hop ABER for 4-QAM modulation [13, eq. (8.112)], we get

$$
P_{4-\mathrm{QAM}}^{\mathrm{DAF}}(\bar{\gamma}, m=1, H) \cong \frac{1}{2}\left(1-\left(\frac{\bar{\gamma}}{1+\bar{\gamma}}\right)^{H / 2}\right) \text {. }
$$

Fig. 6 shows the end-to-end ABER $\left(P_{H}\right)$ (both theoretical and simulation results) as a function of the single-hop average SNR per bit $(\bar{\gamma})$ for $H=2$ with BDPSK, $H=2$ with 4-QAM, and $H=100$ with 4-QAM using both DAF and AAF relaying. For $H=2$, note that DAF relaying barely outperforms AAF for low SNR with both modulations. The difference is less marked with the coherent 4-QAM receiver than with the noncoherent DBPSK receiver. However, regardless the modulation scheme, both DAF and AAF performance tend to equalise as SNR increases. To have a comparison baseline for the general case of an arbitrary number of hops, we also plot the Lower Bound for the AAF relaying.

For $H=100$ and 4-QAM, the Lower Bound for $P_{H}$ with AAF relaying suggests a potential gain of AAF over DAF. However, simulation results for AAF (squares joined with dotted line) dispel the illusion of any such gain. Nevertheless, the gain obtained from DAF over AAF is minimal. The experimental results for AAF with $H=100$ are consistent with the results for AAF, and $H=2$ (either theoretical or simulation results) and their respective lower bounds. 


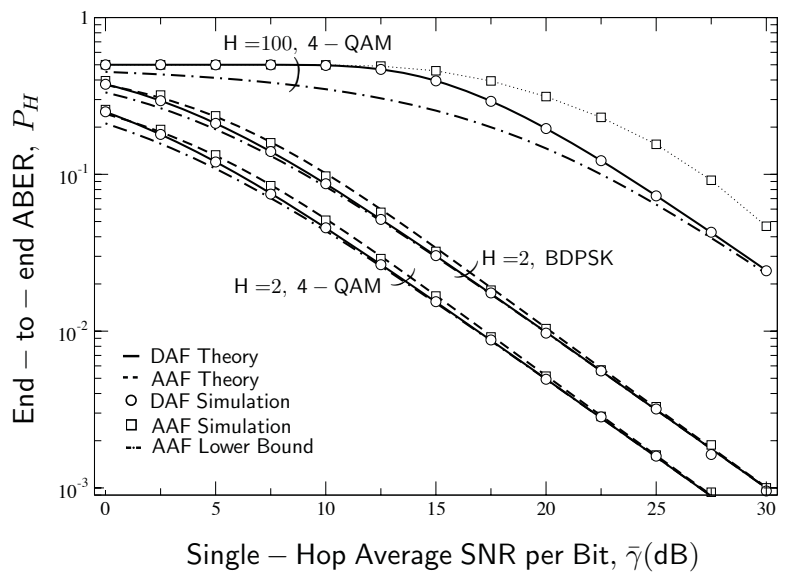

Fig. 6. End-to-end ABER for routes over Rayleigh fading channels with identical statistical behaviour. Three cases are presented: $H=2$ with BDPSK, $H=2$ with 4 -QAM, and $H=100$ with 4-QAM. Continuous lines correspond to $P_{H}$ for DAF relaying (24) and (25). Dashed lines correspond to $P_{H}$ for AAF relaying (19) and (20). Dash-dotted lines correspond to the Lower Bound of $P_{H}$ for AAF relaying (22) and (23). Simulation results for both DAF (circles) and AAF (squares) strategies are shown. The dotted line for the AAF simulations in the $H=100$ case is only shown as a visual aid.

As opposed to what happens in cooperative communications [18], we conclude that, in single-route (non-cooperative communication) MHWN with Rayleigh fading channels, DAF relaying provides a higher performance than AAF relaying.

Returning to the motivation of this section, determining whether DAF or AAF are preferable for multihop relaying, from the previous results for Rayleigh fading channels, it would be apparent that DAF is only slightly more advantageous. However, in the general case of Nakagami- $m$ relay channels $(m>1)$ and a number of relays higher than one, the advantage of DAF over AAF becomes quite relevant.

In the upper half of Fig. 7, we show the end-to-end ABER (theoretical and simulation, where the correctness of the theoretical expressions is apparent from the comparison with the simulation results) as a function of the SNR per bit for $H=2$ with 4-QAM modulation, using both DAF and AAF relaying, over Nakagami- $m$ channels with $m \in\{2,3,4\}$. Analogous to the Rayleigh channel case, in Nakagami- $m$ propagation channels the DAF relaying barely outperforms AAF for low SNR values. However, as index $m$ increases, for a fixed value of SNR, the difference in end-to-end ABER between the DAF and AAF strategies increases.

However, the situation drastically changes as the number of relays increases. In the lower half of Fig. 7 the end-to-end ABER for both the DAF and AAF strategies with $H=100$ is shown. In this case, the only possible comparison with AAF is from simulation results (squares joined with dotted lines) because of the lack of even a Lower Bound similar to (21) and applicable for Nakagami- $m$ channels. In this case, the disparity in the performance of DAF and AAF is striking, and it grows with the $m$-index. The difference between DAF and AAF end-to-end ABER ranges from 2 orders of magnitude (Nakagami-2) to 4 orders of magnitude in the Nakagami-4 fading case for $\bar{\gamma}=20 \mathrm{~dB}$. So, the DAF end-to-end ABER

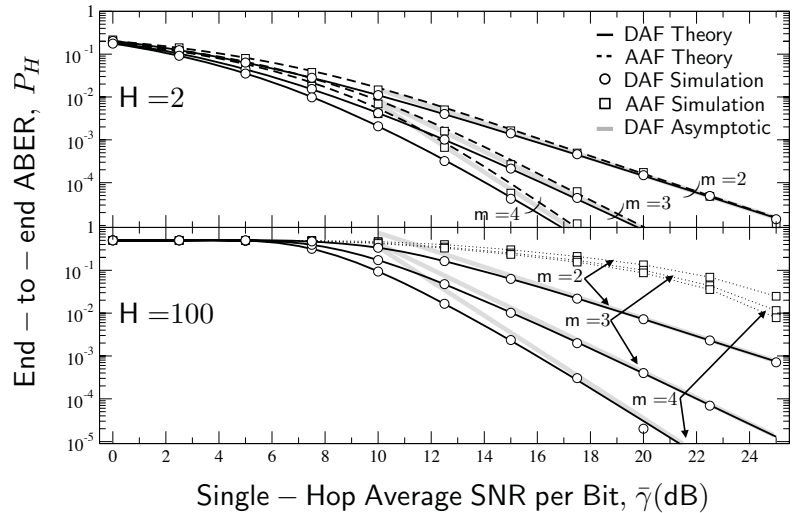

Fig. 7. End-to-end ABER for routes of $H=2$ hops (top) and $H=$ 100 (bottom) over Nakagami- $m$ (with $m=2,3$ and 4) fading channels and 4-QAM modulation with identical statistical behaviour for all the hops. Continuous lines correspond to the exact $P_{H}$ for DAF relaying (18). Short dashed lines correspond to the exact $P_{H}$ for AAF relaying (14). Thick grey lines correspond to the asymptotic expression of $P_{H}$ with the DAF strategy (32) (for the sake of clarity, only the region of $\bar{\gamma} \geq 10 \mathrm{~dB}$ is plotted). Simulation results for different Nakagami- $m$ channels for both DAF (circles) and AAF (squares) strategies are shown. The dotted lines for AAF simulations with $H=100$ are only shown as a visual aid.

decreases exponentially with a greater exponent than the AAF end-to-end ABER, at least in the range of average SNR per bit considered in these simulations. To answer whether this behaviour is expected to hold in the asymptotic limit $(\bar{\gamma} \rightarrow \infty)$ for a generic Nakagami- $m$ channel, we analyze the asymptotic behaviour of the end-to-end ABER for an arbitrary number of relays in the following subsection.

\section{B. Asymptotics of End-to-End ABER with Nakagami-m Fad- ing}

In order to verify whether the advantage of DAF over AAF in Nakagami- $m$ relay channels will hold in the whole range of SNR, we analyze now the asymptotic behaviour of the endto-end ABER.

In the context of wireless communications, at high SNR, the ABER is commonly expressed as a function of the average SNR per bit $\bar{\gamma}$ as $P(\bar{\gamma}) \propto \bar{\gamma}^{-\alpha}$, where $\alpha$ represents the diversity gain [19], [20]. So, parameter $\alpha$ corresponds to the slope of the $P(\bar{\gamma})$ versus $\bar{\gamma}$, at high SNR, in a log-log scale. Thus the following analysis is centered in the calculation of the diversity gain with the use of AAF and DAF as a comparison between both strategies.

To obtain the diversity gain of both AAF and DAF strategies, we will examine the asymptotic behaviour of their respective end-to-end ABER using the closed-form expressions derived above. The asymptotic expansion in (14) and (18) for large enough SNR provides the diversity gain as the lowest (non-zero) absolute value exponent of $\bar{\gamma}$. To get these expansions, we will take into account the main terms of the asymptotic expansion of the Generalized Hypergeometric 
Function. Using the method shown in [21], we obtain

$$
\begin{gathered}
{ }_{p} F_{q}\left(a_{1}, a_{2}, \ldots, a_{p} ; b_{1}, b_{2}, \ldots, b_{q} ; z\right) \approx \\
\approx \lambda_{1}(-z)^{-a_{1}}+\lambda_{2}(-z)^{-a_{2}}+\cdots+\lambda_{p}(-z)^{-a_{p}} ; \\
|z| \rightarrow \infty, \forall a_{i} \neq a_{j}, i \neq j
\end{gathered}
$$

where $\lambda_{i}$, with $i \in\{1,2, \ldots, p\}$ are constants defined as follows:

$$
\lambda_{i}=\frac{\prod_{j=1}^{q} \Gamma\left(b_{j}\right) \prod_{k \neq i}^{p} \Gamma\left(a_{k}-a_{i}\right)}{\prod_{n \neq i}^{p} \Gamma\left(a_{n}\right) \prod_{l=1}^{q} \Gamma\left(b_{l}-a_{i}\right)}+O\left(\frac{1}{z}\right) .
$$

Using (26), the asymptotic expansion of (14) is given by

$$
\begin{aligned}
& P_{4-\mathrm{QAM}}^{\mathrm{AAF}}(\bar{\gamma}, m, H=2) \approx \frac{1}{2}-\frac{2^{1-8 m} \pi^{3 / 2} \Gamma(4 m)}{\Gamma^{3}(m)} \times \\
& \times\left(\lambda_{1}\left(\frac{\bar{\gamma}}{m}\right)^{-m}+\lambda_{2}\left(\frac{\bar{\gamma}}{m}\right)^{-2 m}+\lambda_{3}\right) ; \bar{\gamma} \rightarrow \infty
\end{aligned}
$$

and, after rewriting (28), we get the asymptotic expansion of (14) to the highest relevant power as

$$
\begin{gathered}
P_{4-\mathrm{QAM}}^{\mathrm{AAF}}(\bar{\gamma}, m, H=2) \approx \\
\approx \frac{\Gamma(m+1 / 2)}{\Gamma(m+2) \sqrt{\pi}}(m+1)\left(\frac{\bar{\gamma}}{m}\right)^{-m}+O\left(\left(\frac{\bar{\gamma}}{m}\right)^{-2 m}\right) ; \\
\bar{\gamma} \rightarrow \infty .
\end{gathered}
$$

Thus, we have analytically proved that the diversity gain of the 2-Hop AAF strategy with 4-QAM modulation in Nakagami- $m$ fading channels is $\alpha=m$.

As we mentioned in the previous section, there is no known exact analytical result for the case of multihop $(H>2)$ AAF in Nakagami- $m$ channels. We must resort to the Lower bound in the Rayleigh channel (Nakagami-1) and 4-QAM modulation, and extract the asymptotic behaviour of the Lower Bound of the end-to-end ABER (23).

$$
P_{4-\mathrm{QAM}}^{\mathrm{AAF}}(\bar{\gamma}, m=1, H) \gtrsim \frac{H}{4} \bar{\gamma}^{-1}+O\left(\bar{\gamma}^{-2}\right) ; \bar{\gamma} \rightarrow \infty \text {. }
$$

Thus, for an arbitrary number of relays and Nakagami- $m$ fading, with $m=1$ (Rayleigh), the diversity gain has an upper bound of $\alpha \leq 1$.

We now obtain the asymptotic behaviour of the arbitrary number of relays suffering from Nakagami- $m$ fading for the DAF case. We start from the asymptotic expansion of the Gaussian Hypergeometric Function [21]

$$
{ }_{2} F_{1}(a, b ; c ; z) \propto 1+\frac{a b}{c} z+\frac{a(1+a) b(1+b)}{2 c(1+c)} z^{2}+O\left(z^{3}\right)
$$

to obtain the asymptotic expansion of (18) as

$$
\begin{gathered}
P_{4-\mathrm{QAM}}^{\mathrm{DAF}}(\bar{\gamma}, m, H) \approx \\
\approx \frac{H}{2} \frac{\Gamma(m+1 / 2)}{\Gamma(m+2) \sqrt{\pi}}(m+1)\left(\frac{\bar{\gamma}}{m}\right)^{-m}+O\left(\left(\frac{\bar{\gamma}}{m}\right)^{-2 m}\right) ; \\
\bar{\gamma} \rightarrow \infty .
\end{gathered}
$$

From this expression, we conclude that the diversity gain of the $H$-Hop DAF strategy is also $\alpha=m$. Fig. 7 shows the asymptotic expression (32) for $m=2,3$ and 4 with $H=2$ and 100. It can be observed the consistency of (18) and (32) for high SNR. Moreover, the diversity gain $\alpha=m$ fits in with the slope of the curve representing (32).
From (29), (30) and (32), we conclude that the same asymptotic behavior of the end-to-end ABER for both the AAF and DAF strategies can be found in a multihop route with Nakagami- $m$ fading. This analytical result is in accordance with the intuition that, for large enough SNR, the performance of both strategies are equal. However, for practical values of SNR (from 5 to $25 \mathrm{~dB}$ ) and from the results presented both in Fig. 6 and Fig. 7, we can see that the DAF strategy is more advantageous than AAF in terms of end-to-end ABER performance. From an intuitive point of view, symbol regeneration with AAF recovers the modulus of the symbol, but it does not recover its phase. On the other hand, DAF regeneration tries to recover both the original modulus and phase. Thus, relaying at low SNR (responsible of a high variability of the phase) is prone to generate more errors with AAF strategy than with DAF.

It is interesting to address the case of the asymptotic performance of many different Nakagami- $m$ channels. In this case, an intuitive estimate is that the link with the worst fading (lowest $m$ ) will determine the asymptotic performance of the end-to-end ABER. If we study the asymptotic limit where the approximation (31) is valid, for a route with $H$ consecutive different Nakagami- $m$ channels, we can write the end-to-end ABER as

$$
\begin{aligned}
& P(H) \approx \sum_{i=1}^{H}\left(\left(\frac{\bar{\gamma}}{m_{i}}\right)^{-m_{i}} \frac{\left.\Gamma\left(m_{i}+1 / 2\right)\right)}{\Gamma\left(m_{i}+2\right) \sqrt{\pi}}\left(m_{i}+1\right) \times\right. \\
\times & \left.\prod_{j=i+1}^{H}\left(1-2\left(\frac{\bar{\gamma}}{m_{j}}\right)^{-m_{j}} \frac{\left.\Gamma\left(m_{j}+1 / 2\right)\right)}{\Gamma\left(m_{j}+2\right) \sqrt{\pi}}\left(m_{j}+1\right)\right)\right)
\end{aligned}
$$

where $m_{i}$ characterizes the $i$-th Nakagami- $m$ fading channel and $i=\{1, \ldots, H\}$. As we have proved in Section II-C, $P(H)$ is invariant to the order of the channels, so we can assume that $m_{1}$ is the lowest Nakagami coefficient, $m_{2}$ the second lowest, etc... Then, the first order approximation of the previous equation can be written as

$$
\begin{gathered}
P(H) \approx \\
\approx\left(\frac{\bar{\gamma}}{m_{1}}\right)^{-m_{1}} \frac{\left.\Gamma\left(m_{1}+1 / 2\right)\right)}{\Gamma\left(m_{1}+2\right) \sqrt{\pi}}\left(m_{1}+1\right)+O\left(\left(\frac{\bar{\gamma}}{m_{2}}\right)^{-m_{2}}\right)
\end{gathered}
$$

Thus, the asymptotic performance of the heterogeneous Nakagami- $m$ route is mainly determined by the diversity gain $\left(m_{1}\right)$ of the link suffering the worst fading, confirming that our previous intuition was correct.

\section{CONCLusions}

In this work we have presented, from an statistical point of view and with no approximations, an analytical expression for the end-to-end ABER in MHWN with DAF relaying. This equation is valid for an arbitrary number of relays, as well as for any modulation and fading channel. We also have derived simple expressions for the very general case of Nakagami$m$ fading. We have derived closed analytical expressions for the end-to-end ABER for both non-coherent (DBPSK) and coherent (4-PSK) modulations (easily generalizable for any 
modulation scheme) for an arbitrary number of relays using the DAF strategy and suffering from Nakagami- $m$ fading. We have also derived closed analytical expressions for the end-toend ABER for the aforementioned conditions for the case of a single relay using the AAF strategy. Furthermore, to determine the comparative performance of both strategies in the whole SNR range, we have produced closed expressions for the asymptotic behaviour of the systems characterized above. With these results, we have shown analytically (and corroborated through simulations) that DAF strategy is advantageous over $\mathrm{AAF}$ in channels with both an increasing number of relays and $m$-index, for working values of SNR.

In a future work, this closed and general formulation for the end-to-end ABER will permit the analysis of a scenario where the distance between source and destination is given to obtain the optimal number of hops and the combination of strategies (modulation and forwarding schemes) that it should be used. This is a optimization problem subject to constraints like total transmission power, interference or routing protocol.

\section{APPENDIXES}

\section{A. Proof of Theorem 1}

Let us consider the following recurrence relation to find the end-to-end ABER after an arbitrary number of hops $H \geq 1$

$$
P_{H}=\left(1-P_{H-1}\right) P(H)+P_{H-1}(1-P(H))
$$

subject to the initial condition $P_{0}=0$. Let us also assume that the statistical characterization of the links provide the same single-hop ABER for all links, i.e,

$$
P(h)=P_{\text {hop }}, \forall h \in 1, \cdots, H
$$

where $P_{\text {hop }}$ is a positive constant in the interval $(0,0.5]$.

To solve (35) we will follow the approach found in [22], similar to one of the procedures to solve linear ordinary differential equations. Following [22], we first transform (35) into an homogeneous linear recurrence relation. Thus, taking into account that

$$
P_{H-1}=\left(1-P_{H-2}\right) P_{\text {hop }}+P_{H-2}\left(1-P_{\text {hop }}\right)
$$

and replacing (37) in (35), we have that

$$
P_{H}-2\left(1-P_{\text {hop }}\right) P_{H-1}+\left(1-2 P_{\text {hop }}\right) P_{H-2}=0 .
$$

Next, the above recurrence relation is converted into the polynomial in $r$

$$
r^{2}-2\left(1-P_{\text {hop }}\right) r+\left(1-2 P_{\text {hop }}\right)=0
$$

where the term $P_{H-k}$ has been replaced by $r^{2-k}$. Solving this polynomial gives two values of $r, r_{1}=1$ and $r_{2}=1-$ $2 P_{\text {hop }}$, and the general solution can be expressed as a linear combination of particular solutions

$$
\begin{aligned}
P_{k} & =c_{1} r_{1}^{k}+c_{2} r_{2}^{k}= \\
& =c_{1}+c_{2}\left(1-2 P_{\mathrm{hop}}\right)^{k}
\end{aligned}
$$

where $c_{1}$ and $c_{2}$ are constant real numbers, whose values are determined by the initial condition.
Thus, for $k=0$ and $k=1$ we have that

$$
\begin{aligned}
& k=0 \quad \rightarrow \quad P_{0}=c_{1}+c_{2} \\
& k=1 \quad \rightarrow \quad P_{1}=c_{1}+c_{2}\left(1-2 P_{\text {hop }}\right) .
\end{aligned}
$$

Solving this system, we obtain that $c_{1}=1 / 2$ and $c_{2}=-1 / 2$, and the general solution for $H$ hops can be expressed as

$$
P_{H}=\frac{1}{2}\left(1-\left(1-2 P_{\text {hop }}\right)^{H}\right), \forall H \geq 0 .
$$

\section{B. Proof of Theorem 2}

When the single-hop ABER cannot be considered equal for all links, the linear recurrence relation given in (1) has no constant coefficients and it cannot be solved using the technique in the previous Appendix. Instead, to solve this variable coefficient recurrence relation we will take the approach provided by [23].

The first step is to rewrite the recurrence relation (1) in the general form

$$
a(H) P_{H}=b(H) P_{H-1}+g(H) .
$$

Rearranging terms in (1), we get

$$
P_{H}=(1-2 P(H)) P_{H-1}+P(H), \forall H \geq 1
$$

and, identifying coefficients in (43) and (44), we have that $a(H)=1, b(H)=1-2 P(H)$, and $g(H)=P(H)$. To reduce (44) to a summation and solve the recurrence, we multiply both sides of (44) by the factor $F(H)$, given by

$$
F(H)=\frac{\prod_{i=1}^{H-1} a(i)}{\prod_{j=1}^{H} b(j)}=\frac{1}{\prod_{j=1}^{H}(1-2 P(j))} .
$$

This way, the recurrence (44) becomes

$$
y(H)=y(H-1)+F(H) P(H)
$$

where $y(H)=F(H+1)(1-2 P(H+1)) P_{H}$. Rewriting recurrence (46) as a sum

$$
y(H)=y(0)+\sum_{i=1}^{H} F(i) P(i)
$$

allows us to express $P_{H}$ also as a sum

$$
\begin{aligned}
P_{H} & =\frac{y(H)}{F(H+1)(1-2 P(H+1))}= \\
& =\frac{y(0)+\sum_{i=1}^{H} F(i) P(i)}{F(H+1)(1-2 P(H+1))}
\end{aligned}
$$

where $y(0)=0$ by the initial condition $P_{0}=0$. According to this and taking into account (45), after some rearrangement we have that (48) can be expressed as

$$
\begin{aligned}
P_{H} & =\prod_{k=1}^{H}(1-2 P(k))\left[\sum_{i=1}^{H} \frac{P(i)}{\prod_{j=1}^{i}(1-2 P(j))}\right]= \\
& =\sum_{i=1}^{H}\left(P(i) \prod_{j=i+1}^{H}(1-2 P(j))\right)
\end{aligned}
$$

which is the solution to recurrence relation (44) with variable coefficients. 
It can be shown that solution given by (49) reduces to that provided by (42) when constant coefficients are considered. Thus, assuming the same value $P_{\text {hop }}$ for the single-hop ABER in all the $H$ links, we can express (49) as

$$
\begin{aligned}
P_{H} & =P_{\text {hop }} \sum_{i=1}^{H} \prod_{j=i+1}^{H}\left(1-2 P_{\text {hop }}\right)= \\
& =P_{\text {hop }} \sum_{i=1}^{H}\left(1-2 P_{\text {hop }}\right)^{H-i}
\end{aligned}
$$

where the last sum is a geometric progression with common ratio $r=1-2 P_{\text {hop. }}$. Since $0<P_{\text {hop }} \leq 0.5$, then $|r|<1$ and the sum converges to $\frac{1-\left(1-2 P_{\text {hop }}\right)^{H}}{2 P_{\text {hop }}}$. This way, we have that

$$
P_{H}=\frac{1-\left(1-2 P_{\mathrm{hop}}\right)^{H}}{2}
$$

which is the same expression as the solution provided in the previous Appendix in 42.

\section{Proof of Corollary 1}

Let us define vector $\mathbf{P}_{H}$ as a row vector of $H$ elements, where element in the $i-t h$ position corresponds to the singlehop ABER in the $i-t h$ hop. This is,

$$
\mathbf{P}_{H}=[P(1), P(2), \cdots, P(H)] .
$$

We have proved in the previous Appendix that the end-toend ABER in a $H$-hop route can be expressed in terms of the $H$ single-hop ABER of the route as

$$
P_{H}=\sum_{i=1}^{H}\left(P(i) \prod_{j=i+1}^{H}(1-2 P(j))\right) .
$$

Expanding terms of (53) for different values of $H$, it is easy to check that (53) can be written as

$$
P_{H}=\sum_{i=1}^{H}(-2)^{i-1} \sum_{m=1}^{C_{i}^{H}} \Lambda_{i, m}^{H}=\sum_{i=1}^{H}(-2)^{i-1} S_{i}^{H}
$$

where $C_{i}^{H}=\left(\begin{array}{c}H \\ i\end{array}\right)$ is the number of possible combinations of $i$ elements taken from vector $\mathbf{P}_{H} ; \Lambda_{i, m}^{H}$ denotes the product of all terms involved in the $m-t h$ combination, and $S_{i}^{H}$ is the sum of $\Lambda_{i, m}^{H}$ from $m=1$ to $m=C_{i}^{H}$.

Now, we define $\mathbf{P}_{H}^{\prime}$ as a random permutation of elements in $\mathbf{P}_{H}$, i.e.,

$$
\mathbf{P}_{H}^{\prime}=\left[P^{\prime}(1), P^{\prime}(2), \cdots, P^{\prime}(H)\right] .
$$

For example, for a route with $H=4$ hops where $\mathbf{P}_{H}=$ $[P(1), P(2), P(3), P(4)]$, a random permutation might provide vector $\mathbf{P}_{H}^{\prime}=[P(3), P(1), P(2), P(4)]$. For a general case, with the new sequence of single-hop ABER $P^{\prime}(i)$, with $i=1, \ldots, H$, the end-to-end ABER is given by

$$
\begin{aligned}
P_{H}^{\prime} & =\sum_{i=1}^{H}\left(P^{\prime}(i) \prod_{j=i+1}^{H}\left(1-2 P^{\prime}(j)\right)\right)= \\
& =\sum_{i=1}^{H}(-2)^{i-1} \sum_{m=1}^{C_{i}^{\prime H}} \Lambda_{i, m}^{\prime H}=\sum_{i=1}^{H}(-2)^{i-1} S_{i}^{\prime H}
\end{aligned}
$$

where $C_{i}^{\prime} H=\left(\begin{array}{c}H \\ i\end{array}\right)$ is the number of possible combinations of $i$ elements taken from vector $\mathbf{P}_{H}^{\prime} ; \Lambda_{i, m}^{\prime H}$ denotes the product of all terms involved in the $m-t h$ combination, and $S_{i}^{\prime}{ }^{H}$ is the sum of $\Lambda_{i, m}^{\prime H}$ from $m=1$ to $m=C_{i}^{\prime} H$.

According to [24], to prove that $P_{H}=P_{H}^{\prime}$, it suffices to demonstrate that $S_{i}^{H}$ is an invariant sum under permutations of elements in vector $\mathbf{P}_{H}$. Note that, depending of the new sequence of single-hop ABER, the particular element $\Lambda_{i, m}^{H}$ in (54) can be different of element $\Lambda_{i, m}^{\prime} H$ in (56). However, note also that the sum of all the $\left(\begin{array}{c}H \\ i\end{array}\right)$ combinations of $i$ elements in vector $\mathbf{P}_{H}$ does not change when elements in $\mathbf{P}_{H}$ are rearranged to have $\mathbf{P}_{H}^{\prime}$. Since $S_{i}^{\prime} H$ encompasses all combinations of the same $H$ elements of $\mathbf{P}_{H}$ (taking $i$ at a time), we conclude that $S_{i}^{H}=S_{i}^{\prime}{ }^{H}$ and, therefore, the end-toend ABER given by (53) is invariant under permutations of the single-hop ABER in the route.

\section{REFERENCES}

[1] G. Farhadi and N. Beaulieu, "Connectivity and bit error rate analysis of mobile ad hoc wireless networks," in Proc. IEEE 64th Vehicular Technology Conf., Sep. 2006, pp. 1-5.

[2] G. Ferrari and O. Tonguz, "Impact of mobility on the BER performance of ad hoc wireless networks," IEEE Trans. Veh. Technol., vol. 56, pp. 271-286, Jan. 2007.

[3] A. Stefanov and E. Erkip, "Cooperative coding for wireless networks," IEEE Trans. Commun., vol. 52, pp. 1470-1476, Sep. 2004.

[4] J. N. Laneman and G. W. Wornell, "Distributed space-time-coded protocols for exploiting cooperative diversity in wireless networks," IEEE Trans. Inf. Theory, vol. 49, no. 10, pp. 2415-2425, Oct. 2003.

[5] M. Haenggi, "On routing in random Rayleigh fading networks," IEEE Trans. Wireless Commun., vol. 4, pp. 1553-1562, July 2005.

[6] F. Zhenghua, L. Haiyun, P. Zerfos, L. Songwu, Z. Lixia, and M. Gerla, "The impact of multihop wireless channel on TCP performance," IEEE Trans. Mobile Comput., vol. 4, pp. 209-221, Mar. 2005.

[7] G. Farhadi and N. Beaulieu, "On the outage and error probability of amplify-and-forward multi-hop diversity transmission systems," in Proc. 2008 IEEE Int. Conf. Commun., May 2008, pp. 3748-3754.

[8] Y. Sheng and J. Belfiore, "Towards the optimal amplify-and-forward cooperative diversity scheme," IEEE Trans. Inf. Theory, vol. 53, no. 9, pp. 3114-3126, Sep. 2007.

[9] G. Farhadi and N. Beaulieu, "Selective decode-and-forward relaying scheme for multi-hop diversity transmission systems," in Proc. 2007 IEEE Global Telecommun. Conf., Nov. 2007, pp. 4385-4390.

[10] Y. Zhihang and I. Kim, "Decode-and-forward cooperative networks with multiuser diversity," in Proc. 2006 Military Commun. Conf., Oct. 2006, pp. 1-7.

[11] W. Tairan, A. Cano, G. Giannakis, and J. Laneman, "High-performance cooperative demodulation with decode-and-forward relays," IEEE Trans. Commun., vol. 55, no. 7, pp. 1427-1438, July 2007.

[12] M. Hasna, "Average BER of multihop communication systems over fading channels," in Proc. 10th IEEE Int. Conf. Electron., Circuits Syst., vol. 2, Dec. 2003, pp. 723-726.

[13] M. K. Simon and M. S. Alouini, Digital Communications over Fading Channels, 2nd edition. New York: Wiley-IEEE Press, 2004.

[14] M. Hasna and M. Alouini, "End-to-end performance of transmission systems with relays over Rayleigh-fading channels," IEEE Trans. Wireless Commun., vol. 2, no. 6, pp. 1126-1131, Nov. 2003.

[15] A. Lakhzouri, E. Lohan, I. Saastamoinen, and M. Renfors, "Interference and indoor channel propagation modeling based on gps satellite signal measurements," in Proc. ION GNSS, Sep. 2005, pp. 896-901.

[16] M. Hasna and M. Alouini, "Harmonic mean and end-to-end performance of transmission systems with relays," IEEE Trans. Commun., vol. 52, no. 1, pp. 130-135, Jan. 2004.

[17] I. S. Gradshteyn and I. M. Ryzhik, Table of Integrals, Series and Products. Boston, MA: Academic Press, 1994.

[18] J. Boyer, D. Falconer, and H. Yanikomeroglu, "Cooperative connectivity models for wireless relay networks," IEEE Trans. Wireless Commun., vol. 6, pp. 1992-2000, June 2007.

[19] J. Proakis, Digital Communications, 4th edition. New York: McGrawHill, 2001. 
[20] Z. Wang and G. B. Giannakis, "A simple and general parameterization quantifying performance in fading channels," IEEE Trans. Commun., vol. 51, pp. 1389-1398, Aug. 2003.

[21] A. Erdelyi, Higher Transcendental Functions, 1st edition. McGraw-Hill, 1953, vol. 1.

[22] M. Spiegel, Calculus of Finite Differences and Difference Equations, 1st edition. New York: McGraw-Hill, 1971.

[23] D. Knuth, The Art of Computer Programming: Sorting and Searching, 1st edition. Reading, MA: Addison-Wesley, 1973, vol. 3.

[24] C. W. McArthur, "Series with sums invariant under rearrangement," American Mathematical Monthly, vol. 75, no. 1, pp. 729-731, 1968.

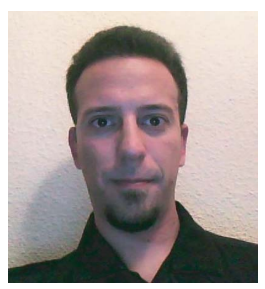

Eduardo Morgado received a degree in telecommunication engineering from the University Carlos III de Madrid, Spain, in 2004 and the PhD degree from the University Rey Juan Carlos, Spain, in 2009. Currently, he is an associate professor in the Department of Signal Theory and Communications, University Rey Juan Carlos, Madrid, Spain. His research interests include signal processing for wireless communications with applications to ad hoc and sensor networks.

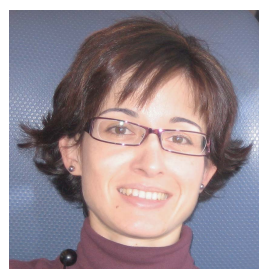

Inmaculada Mora-Jiménez received the Telecommunication engineering degree from Universidad Politcénica de Valencia, Spain, in 1998, and the $\mathrm{PhD}$ degree from University Carlos III de Madrid, Spain, in 2004. Currently, she is an Associate Professor in the Department of Signal Theory and Communications at University Rey Juan Carlos in Madrid, Spain. Her main research interests include statistical learning theory, neural networks, and their applications to image processing, bioengineering, and communications.

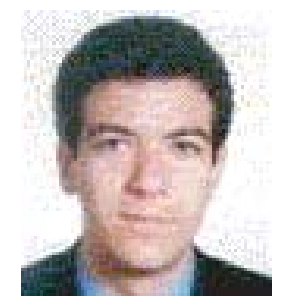

Juan J. Vinagre works in the Signal Theory and Communications department in Rey Juan Carlos University. Dr. Vinagre has considerable commercial experience in the development of large scale communication networks for airports, buildings, and other large enterprises. He has also managed large engineering teams. He has participated in more than 30 research and development projects. His research lines include Wireless Ad Hoc and Sensor networks, routing analysis, and vehicular communications with and traffic optimization. applications to indoor location, energy efficiency,

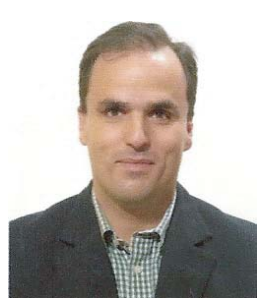

Javier Ramos received the B.Sc and M.Sc. degrees from the Polytechnic University of Madrid, Spain. Between 1992 and 1995 he cooperated in several research projects at Purdue University, Indiana, USA, working in the field of Signal Processing for Communications. He received the $\mathrm{PhD}$ degree on 1995. During 1996 he was Post-Doctoral Research Associate at Purdue University. Dr. Ramos received the Ericsson award to the best Ph.D. dissertation on Mobile Communications in 1996. From 1997 to 2003 Dr Ramos was associate professor at Carlos III University of Madrid. Since 2003 Dr Ramos is the Dean of the Telecommunications Engineering department at the Rey Juan Carlos University of Madrid. His present fields of research are Broadband Wireless Services and Technologies, Wireless Networks Security and distributed sensing.

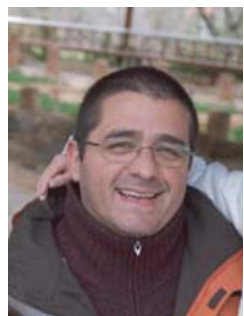

Antonio J. Caamaño received the B.Sc. and Ms.C. degrees in Theoretical Physics in 1995 from the Autonomous University of Madrid and his Ph.D. in Telecommunications Engineering from the Carlos III University of Madrid in 2003. From 2003 he is Associate Professor in the Rey Juan Carlos University of Madrid and since 2005 he is Head of the Signal Theory and Communications Department. $\mathrm{He}$ has directed or cooperated in more than 40 private and publicly-funded research projects. $\mathrm{He}$ has published research results in Field Emission devices for communications, Blind Source Separation, Multiuser Detection and processing of biological signals. His main research interests lie in the field of large-scale MANET optimization, distributed computing and neuromorphic processing. 